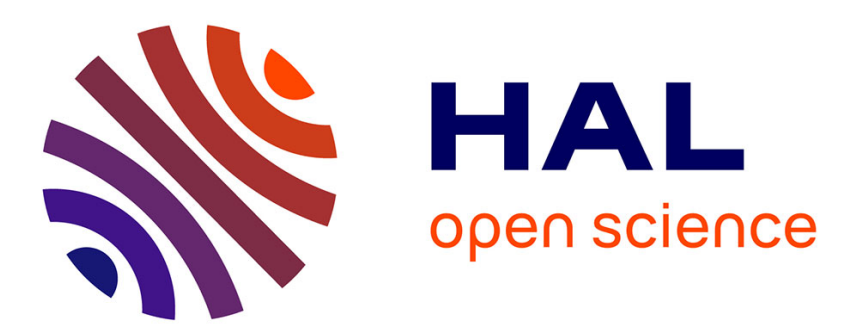

\title{
Molecular modeling study of intercalation complexes of tricyclic carboxamides with d(CCGGCGCCGG) and d(CGCGAATTCGCG)
}

Athanasia Varvaresou, Kriton Iakovou

\section{- To cite this version:}

Athanasia Varvaresou, Kriton Iakovou. Molecular modeling study of intercalation complexes of tricyclic carboxamides with d(CCGGCGCCGG) and d(CGCGAATTCGCG). Journal of Molecular Modeling, 2010, 17 (8), pp.2041-2050. 10.1007/s00894-010-0891-5 . hal-00651659

\author{
HAL Id: hal-00651659 \\ https://hal.science/hal-00651659
}

Submitted on 14 Dec 2011

HAL is a multi-disciplinary open access archive for the deposit and dissemination of scientific research documents, whether they are published or not. The documents may come from teaching and research institutions in France or abroad, or from public or private research centers.
L'archive ouverte pluridisciplinaire HAL, est destinée au dépôt et à la diffusion de documents scientifiques de niveau recherche, publiés ou non, émanant des établissements d'enseignement et de recherche français ou étrangers, des laboratoires publics ou privés. 


\title{
Molecular modeling study of intercalation complexes of tricyclic carboxamides with d(CCGGCGCCGG) $)_{2}$ and d(CGCGAATTCGCG)
}

Received: 30.05.2010 / Accepted: 19.10.2010

Athanasia Varvaresou ${ }^{1, \bowtie}$, and Kriton Iakovou ${ }^{2, \dagger}$

${ }^{1}$ Laboratory of Cosmetology, Department of Aesthetics and Cosmetology, Technological Educational Institution of Athens, Ag. Spyridona, Egaleo 12 210, Athens, Greece

${ }^{2}$ Ministry of Health and Social Solidarity, Aristotelous 17, 10433 Athens, Greece

Tel.:+30 2106810354; Fax: +30 2106810359; Ious 7, 14563 Kifisia, Athens, Greece; Email: varvaresou@pharm.uoa.gr

\begin{abstract}
Tricyclic dyes with different mesoatoms such as xanthenes (fluorescein, eosin) anthracenes and acridines (proflavine) approved by the Food and Drug Administration (FDA) for use in foods, pharmaceuticals and cosmetic preparations interact with DNA, and some of them do so through intercalation. Hyperchem 7.5, Spartan 04, Yasara 10.5.14 program packages and molecular modeling, molecular mechanics and dynamics techniques with the oligonucleotides d(CCGGCGCCGG)2 and d(CGCGAATTCGCG)2 were utilized in order to examine the mode of binding to DNA of a range of tricyclic carboxamides bearing $\mathrm{N}, \mathrm{N}$ dimethylaminoethyl side chain i.e. 9-amino-DACA, anthracene, acridine-1-carboxamide, acridine-4-carboxamide (DACA), azacridine, phenazine, pyridoquinoxaline, oxopyridoquinoxaline, phenoxazine and xanthenone or N,N-dimethylaminobutyl moiety i.e. phenazine and acridine. The bicyclic quinoline-8-carboxamide was also examined for comparison reasons. On the basis of our data, prerequisite for the interaction between protonated $\mathrm{N}, \mathrm{N}$-dimethylaminoethyl moiety and guanine is the formation of only one internal hydrogen bond between carboxamide and peri $\mathrm{NH}+$ in the case of 9-amino-DACA or peri $\mathrm{N}$ in the cases of DACA, azacridine, phenazine and pyridoquinoxaline. The presence of an additional internal hydrogen bond between oxygen carboxamide and protonated N,N-
\end{abstract}


dimethylamino group in the cases of tricyclic systems bearing peri $\mathrm{NH}$ (phenoxazine) or $\mathrm{O}$ (xanthenone) group, prevents the interaction between side chain and guanine. Also, the formation of one internal hydrogen bond between oxygen carboxamide and protonated N,Ndimethylamino group inhibits the interaction between side chain and guanine in the case of acridine-1-carboxamide. Our findings are in accordance with previously reported results obtained from the kinetic studies of the binding of acridine and related tricyclic carboxamides to DNA.

Keywords Tricyclic carboxamides - DNA intercalation ' Molecular mechanics • Molecular dynamic simulations 


\section{Introduction}

Tricyclic dyes with different mesoatoms such as xanthenes (fluorescein, eosin) anthracenes and acridines (proflavine) approved by the Food and Drug Administration (FDA) for use in foods, pharmaceuticals and cosmetic preparations interact with DNA, and some of them do so through intercalation [1-4].

The intercalation process reflects the ability of a planar aromatic or heteroaromatic system to become inserted between adjacent base pairs of a DNA molecule without disturbing the overall stacking pattern [5].

The acridine-4-carboxamides are a series of DNA intercalating topoisomerase poisons, developed by Denny and colleagues, that show remarkable variation in antitumor activity for minimal changes in molecular structure [6]. Some are potent topoisomerase poisons with widespread antitumor efficacy, for example, N-[2-(dimethylamino)ethyl]acridine-4carboxamide (DACA), which is a DNA-intercalating agent capable of inhibiting both topoisomerases I and II [7-10] and is in phase II clinical trial. For the 9-aminoacridine class of these compounds, the parent of which is 9-amino-DACA, there are tight correlations between ligand structure, cytotoxicity and DNA-binding kinetics [11-15]. Some of these compounds have been determined by X-ray crystallography. In the crystal structures of 9-amino-DACA and its 5-fluoro derivative bound to the hexanucleotide d(CGTACG $)_{2}[16,17]$, and of 6-Br-9amino-DACA bound to the brominated hexanucleotide $\mathrm{d}\left(\mathrm{CG}^{5 \mathrm{Br}} \mathrm{UACG}\right)_{2}$ [18], the 4carboxamide group lies in the plane of the chromophore, and the carbonyl oxygen atom forms an internal hydrogen bond with the protonated N10 nitrogen of the acridine ring. The side chain lies in the DNA major groove with its protonated $N, N$-dimethylamino group forming hydrogen-bonding interactions with the $\mathrm{O} 6$ and N7 atoms of guanine G2. In each case, a hydrogen-bonded water molecule bridges the $\mathrm{NH}$ of the carboxamide group to the guanine $\mathrm{G} 2$ phosphate at the intercalation site. These structures have provided a molecular rationale for understanding the structure-activity relationships for antitumor activity and enabled a mechanistic interpretation of the dependence of kinetics on ligand structure [16, 17]. In particular, they have permitted the critical step in the dissociation kinetics profile that correlates with cytotoxicity and antitumor activity to be identified with the side chain-guanine interaction [15-17]. Attempts to crystallize DACA, its phenazine analogue and related agents with $\mathrm{d}(\mathrm{CGTACG})_{2}$ have yielded an unusual quadruplex which does not add to our 
understanding of their complexes with duplex DNA [19-21], and other DNA sequences have failed to give diffracting crystals.

The most significant features of the structure-activity relationships are that a nitrogen atom must be peri to the carboxamide, that the carboxamide must have an unsubstituted $\mathrm{NH}$ group, and that there must be two methylene groups between the carboxamide $\mathrm{NH}$ and the terminal protonated $N, N$-dimethylamino group $[11,16]$. To the best of our knowledge there are only limited examples in the recent bibliography regarding the exploration of the energy consequences between intercalative ligands and oligonucleotides. Theoretical investigation of the $\pi-\pi$ interactions between rhodomyrtoxin B and the guanine-cytosine base pair by molecular mechanics and ab initio molecular-orbital techniques is referred by Setzer $\mathrm{W}$ et al [22], whereas the intermolecular interaction between alkaloids such stauranthine and skimmianine and guanine-cytosine by ab initio molecular techniques has been also reported by Byler K. et al [23]. Furthermore, semiempirical calculations carried out by Loza-Mejía et al regarding the interaction between 9-anilinoacridines, 9-anilinothiazolo[5,4-b]quinolines and 9-anilinoimidazo[4,5-b]quinolines and selected hexanucleotides revealed the strong influence of the replacement of the benzene moiety for a heterocyclic ring on the simulation of DNA-intercalator complexes [24]. Also, molecular dynamic techniques were used in order to examine energetic inetractions between antitumor mono- or bisnaphthalimides and $\mathrm{d}(\mathrm{TG})_{2}$ and $\mathrm{d}(\mathrm{ATGCAT})_{2}[25,26]$. Our aim in this molecular modeling study was to discover whether the $N, N$-dimethylaminoethyl or $N, N$-dimethylaminobutyl side chain of a range of intercalated tricyclic carboxamides with neutral chromophores can form hydrogen-bonding interactions with the O6/N7 atoms of guanine in a manner similar to that of 9-amino-DACA. We believe that our findings could be useful for the design of new chromophores with better DNA-binding activity and anti-tumor action. Furthermore, we think it will be interesting to explore the interaction of these chromophores with DNA, since this kind of molecules are used as dyes in many materials (cosmetics, drugs and food) consumed by people.

Thus, in the inadequacy of direct structural information, and in light of some studies of kinetics of dissociation of DNA-ligand complexes and the known similarities in the DNA binding characteristics of DACA and 9-amino-DACA, we constructed computer models of the complexes of 9-amino-DACA and a range of related linear cyclic carboxamides (compounds 1-12, Fig. 1) $[6,27,28]$ with oligonucleotides d(CCGGCGCCGG) 2 and d(CGCGAATTCGCG) $)_{2}$. We examined for comparison reasons quinoline-8-carboxamide, a 
bicyclic system with $\mathrm{N}$ peri to carboxamide side chain, which is known to bind to DNA in a nonintercalative fashion and is inactive as an antitumor agent (Fig. 1) [29].

Since our results suggested that the side chains of systems which bear a peri nitrogen atom (47) make interactions with DNA in a manner similar to the side chain of 9-amino-DACA (1), we performed also molecular dynamics simulations at $298 \mathrm{~K}$ for $15 \mathrm{~ns}$ for the complexes of compounds 1 and 4-7 with d(CCGGCGCCGG) $)_{2}$ and d(CGCGAATTCGCG $)_{2}$.

\section{Computational methods}

\section{Molecular mechanics and molecular dynamic simulations}

Insight into the mode of binding of compounds 1-13 to DNA was gained through molecular modeling, molecular mechanics and dynamics techniques using the oligomers d(CCGGCGCCGG $)_{2}$ and d(CGCGAATTCGCG $)_{2}$.

The sequence was selected according to the preference for intercalation at 5'-CpG-3' steps exhibited by monointercalating acridine derivatives to DNA and to modeling experience [1517, 30-35] with related intercalating agents.

Ring systems 1-13 were model-built in Hyperchem 7.5 [36] and Spartan 04 program packages [37] using standard geometries, which were fully optimized by means of the program Spartan 04 with Hartree-Fock method and the $6-31 G^{*}$ basis set.

The crystal structures of oligonucleotides $\mathrm{d}(\mathrm{CCGGCGCCGG})_{2} \quad$ (PDB:1cgc) and $\mathrm{d}(\text { CGCGAATTCGCG) })_{2}$ (PDB: 1d30) were downloaded from the Protein Data Bank (PDB) using Yasara 10.5.14 [38]. The molecules of water and ligand were deleted.

Thirty six model complexes were constructed for compounds $\mathbf{1 - 3}$ and $\mathbf{8 - 1 3}$ by inserting the chromophores into the central CG of the d(CCGGCGCCGG) $)_{2}$ in the four possible orientations relative to the base pairs, denoted M1, M2, m1, and m2, as shown in Fig. 2 Two through the major groove: one with the ring bearing the side chain between the central $\mathrm{CG}$ and the other with the ring bearing the side chain between the central GC; and the same orientations leaving the side chain in the minor groove. It is noteworthy that the carboxamide group of compounds 
with peri nitrogen (4-7), can lie in two orientations in the plane of the chromophore, viz, with the carbonyl oxygen pointing toward the peri nitrogen, the cis position, or away, the trans position. In these conformations the resonance stabilization between carboxamide and chromophore is maximized, and in the trans position there is the added possibility that the carboxamide NH group can form an internal hydrogen bond with the peri nitrogen. Using the mechanical program Spartan 04 and the 6-31G* basis set, we calculated that the trans conformation of these compounds is more stable than cis by about $12.5 \mathrm{kcal} \mathrm{mol}^{-1}$.

Therefore, sixteen model complexes were constructed for compounds 4-7 with the carboxamide group in trans orientation and sixteen model complexes were constructed by inserting the chromophores into the $\mathrm{d}(\mathrm{CCGGCGCCGG})_{2}$ with the ring bearing the side chain stacked between the central $\mathrm{CG}$ and the carboxamide groups of the optimized intercalators rotating by $180^{\circ}$ (cis orientation).

Additional thirteen model complexes were constructed for compounds 1-13 by inserting the chromophores into the $\mathrm{d}(\mathrm{CGCGAATTCGCG})_{2}$ through the major groove, with the ring bearing the side chain stacked between the terminal CG (M1) and four model complexes were constructed for compounds 4-7 with the carboxamide groups of the optimized intercalators rotating by $180^{\circ}$ (cis orientation).

Processing of data and simulations of all models (1-13) were done using the program Yasara, with the Yamber 3 force field [39]. Van der Waals pairs cutoff distance was $7.86 \AA$ and particle mesh Ewald (PME) long range electrostatics were employed [40]. Simulation Structures were energy-minimized to remove bumps and correct the covalent geometry. After removal of conformational stress by steepest descent minimization, the procedure continued by simulated annealing (time step $2 \mathrm{fs}$, atom velocities scaled down by 0.9 every $10^{\text {th }}$ step) until convergence was reached, i.e., the energy improved by less than $0.1 \%$ during 200 steps.

Periodic boundary simulations were done on orthorhombic cells of extra extension along each axis of the complex of $5 \AA$. Yasara program assigned force field parameters, filled the cell with water and placed $\mathrm{Na}+$ and $\mathrm{Cl}^{-}$counter ions at the most favorable positions to make the cell neutral. The simulation cell was filled with water to a density of $0.997 \mathrm{~g} / \mathrm{l}$, and the minimization procedure was repeated, first with the water solvent, then with the oligonucleotide, the ligand, and finally with the whole system. Minimization was followed by a short equilibration procedure to $298 \mathrm{~K}$. Resulting minimized and equilibrated models were 
subsequently used for MD simulations. Analysis of data was also done with Yasara, using the distributed computing facility. Data is presented as mean \pm standard deviation.

Trajectories of MD simulations were sampled at intervals of $1 \mathrm{ps}$, for a total of $15 \mathrm{~ns}$ for each model. We analyzed the stability of trajectories to ensure that the models were at equilibrium. The MD trajectories were inspected, also visually, to assess their structural stability. The gyration radius of the models is maintained quite constant throughout all the trajectories (average values of 12.3-12.9 \pm 0.1-0.3 $\AA$ ). The quantitative assessment of the stability of the trajectory has been done by measuring two parameters: the root mean square (RMS) of the complexes, and the average potential energy of the system. These two parameters should not vary considerably during the simulation when the system is at equilibrium. During the first $200 \mathrm{ps}$ there is a change of energy due to the relaxation of the system after assignment of initial velocities. The potential energy for all trajectories converges to a constant average value, demonstrating the reproducibility of the simulations. We considered that the systems got equilibrium after 500 ps equilibration, as they reached mean energy values that were less than $3 \%$ different from the average asymptotic values. Hence, all average values presented were evaluated after the first 700 ps of simulation. The root-mean-square deviation of the complexes of compounds 1 and 4-7 (in trans and cis orientation) with d(CCGGCGCCGG) $)_{2}$ and $\mathrm{d}(\mathrm{CGCGAATTCGCG})_{2}$ with respect to the initial structures were evaluated. The RMSD values ranged from 0.67 to 1.2 (for the cis orientation) \pm 0.1-0.4 $\AA$ (available as supporting information, Fig. 3). The potential energy of the system was defined as the average force field energy of measured after the first 700 ps simulation in all trajectories (available as supporting information, Fig. 4). The potential energy values ranged from -45130 (1) to -34716 [(5) in the cis conformation] $\pm 86-124 \mathrm{kcal} \mathrm{mol}^{-1}$.

\section{Results}

\section{Energy minimization}

Results are summarized in Table 1.

The model compounds studied can be classified with reference to calculated internal hydrogen bonds, as follows: 
A. Compounds 1, 4-8, and 11-13, which have a $-\mathrm{NH}^{+}$group or $-\mathrm{N}$ atom peri to the carboxamide. These may be divided into two types.

1. Compounds 1, and 4-7, which have one internal hydrogen bond between the carboxamide and the $-\mathrm{NH}^{+}$or $-\mathrm{N}$ group in the peri position (compound 7, Fig. 5).

2. Compounds 8 (Fig. 5), 11-13, which have an additional internal hydrogen bond between the carboxamide and the charged amino group.

B. Compounds 2, 3, 9 and 10, which lack a nitrogen peri to the carboxamide. These may be divided into three types.

1. Compound 2, which lacks internal hydrogen bonds (Fig. 5).

2. Compound 3, which has one internal hydrogen bond between the carboxamide and the dimethylammonium group (Fig. 5).

3. Compounds 9, and 10, which have two internal hydrogen bonds : one between the carboxamide and the $-\mathrm{NH}$ or $-\mathrm{O}$ group in the peri position and the other between the carboxamide and the dimethylammonium group.

Schematic view from the major groove of the complexes between compounds 1-13 and $\mathrm{d}(\mathrm{CCGGCGCCGG})_{2}$ in the four possible orientations M1, M2, $\mathrm{m} 1$ and $\mathrm{m} 2$ is shown in Fig. 2.

In the complexes of compounds $\mathbf{1}$ and 4-7 with the oligonucleotide d(CCGGCGCCGG) $)_{2}$ in the four possible orientations, the $N, N$-dimethylammonium group forms strong hydrogen bonding interaction with the G6-N7/O6 atoms, O6 of G16, O2 of C5 and O2 of $\mathrm{C} 15$ for the $\mathrm{M} 1, \mathrm{M} 2, \mathrm{~m} 1$ and $\mathrm{m} 2$ orientations, respectively.

In the four (2)-CCGGCGCCGG complexes, the $N, N$-dimethylammonium group does not form hydrogen bond with the heteroatoms of the base pairs in the M1 orientation, whereas the carboxamide group hydrogen bonds strongly to O6 of G16, N2 of G16 and N3 of G6 for the $\mathrm{M} 2, \mathrm{~m} 1$ and $\mathrm{m} 2$ orientations, respectively.

In the (3)-CCGGCGCCGG complexes the $N, N$-dimethylammonium group does not form hydrogen bond with the base pairs in the M1 orientation, whereas the carboxamide group 
bonds strongly to $\mathrm{N} 3$ of $\mathrm{C} 15$, the sugar $\mathrm{O}^{\prime}$ ' atom of G6 and the sugar O4' atom of G16 for the $\mathrm{M} 2, \mathrm{~m} 1$ and $\mathrm{m} 2$ orientations, respectively.

In the complexes of compounds $\mathbf{8}$ and 11-13 with the decanucleotide d(CCGGCGCCGG) $)_{2}$ in the four possible orientations, the carboxamide and the $N, N$-dimethylammonium groups are not sufficiently close to any electronegative base pair atom to be involved in hydrogen bonding. Additionally, in the M1 orientation the $\mathrm{H}$ atom of the carboxamide bonds with the peri heteroatom of the chromophore and the $\mathrm{O}$ atom of the carboxamide bonds with the $\mathrm{N}, \mathrm{N}$ dimethylammonium group (compounds $\mathbf{1 1}$ and 13)

In the four (9)-CCGGCGCCGG complexes, the $N, N$-dimethylammonium group makes strong hydrogen bonding interaction with the $\mathrm{N} 4$ of $\mathrm{C} 5, \mathrm{~N} 3$ of $\mathrm{G} 6$ and $\mathrm{G} 16$ for the M1, $\mathrm{m} 1$ and $\mathrm{m} 2$ orientations, respectively. On the contrary, there is no hydrogen bonding interaction between $\mathrm{N}, \mathrm{N}$-dimethylammonium group and the base pairs in the $\mathrm{M} 2$ orientation. The $\mathrm{O}$ atom of the carboxamide bonds with the $\mathrm{N}, \mathrm{N}$-dimethylammonium group and with the $\mathrm{H}$ atom of the $\mathrm{NH}$ of the chromophore in the M1 orientation.

In the four (10)-CCGGCGCCGG complexes, the $N, N$-dimethylammonium group hydrogen bonds to $\mathrm{N} 3$ of G6 and makes a weak interaction with the N3 of G16 for the $\mathrm{m} 1$ and $\mathrm{m} 2$ orientations, respectively. In the M1 orientation, there is no hydrogen bonding interaction between $N, N$-dimethylammonium group and the base pairs, whereas there is hydrogen bonding interactions between the $\mathrm{H}$ atom of the carboxamide group and the peri heteroatom of the chromophore and additionally between the $\mathrm{O}$ atom of the carboxamide moiety and the $N, N$-dimethylammonium group.

\section{Molecular dynamic simulations}

In the molecular dynamic simulation of the (1)-CCGGCGCCGG complex, the hydrogen of the $N, N$-dimethylammonium is strongly hydrogen bonded to O6 of guanine- 6 and makes a weak interaction with the N7 of guanine-6 (Fig. 6). In the molecular dynamic simulation of the (1)-CGCGAATTCGCG complex, the hydrogen of the $N, N$-dimethylammonium makes a strong hydrogen bond solely to O6 of guanine-2. The side-chain torsion angle shows a gauche conformation relating the two side-chain nitrogen atoms, just as it occurs in the crystal structures [17]. 
In the molecular dynamic simulation of the (4)-CCGGCGCCGG complex, with the carboxamide group in the cis orientation (the carbonyl oxygen points toward the peri nitrogen), the hydrogen of the $N, N$-dimethylammonium moiety makes strong hydrogen bonding interaction with the O6 of guanine- 6 and feebly interacts with the N7 of the same guanine. In the (4)-CCGGCGCCGG complex, with the carboxamide group in the trans orientation (the carbonyl oxygen points away the peri nitrogen), the hydrogen of the $N, N$ dimethylammonium moiety makes strong hydrogen bonding interaction with the O6 of guanine-6.

In the molecular dynamic simulation of the (4)-CGCGAATTCGCG complex, with the carboxamide group in the cis orientation, the hydrogen of the $N, N$-dimethylammonium moiety makes a strong hydrogen bond solely with the O6 of guanine-2. In the (4)CGCGAATTCGCG complex, with the carboxamide group in the trans orientation, the hydrogen of the $N, N$-dimethylammonium hydrogen strongly bonds to the O6 of guanine-2 and weakly interacts with the N7 of guanine-2.

In the molecular dynamic simulation of the (5)-CCGGCGCCGG complex, with the carboxamide group in the cis orientation, the hydrogen of the $N, N$-dimethylammonium moiety forms a hydrogen bond with the O6 of guanine-6 and is close to G6-N7, with distance $\mathrm{H}(\mathrm{N}) \ldots \mathrm{O}(\mathrm{G})$ of $3.2 \AA \pm 0.3$. In the (5)-CCGGCGCCGG complex, with the carboxamide group in the trans orientation, the protonated $N, N$-dimethylamino group makes a strong hydrogen bond to $\mathrm{O} 6$ of guanine- 6 and weakly interacts with the N7 of guanine-6. In the (5)CGCGAATTCGCG complex, with the carboxamide group in the cis orientation, the protonated $N, N$-dimethylamino group forms a hydrogen bond with O6 of guanine-2. In the (5)-CGCGAATTCGCG complex, with the carboxamide group in the trans orientation, the $\mathrm{N}, \mathrm{N}$-dimethylammonium moiety forms strong hydrogen bonding interaction with $\mathrm{O} 6$ of $\mathrm{G} 2$ and is also in contact of $3.3 \AA \pm 0.2$ with $\mathrm{N} 7$ of G2.

In the molecular dynamic simulation of the (6)-CCGGCGCCGG complex, with the carboxamide group in the cis orientation, the protonated $N, N$-dimethylamino group strongly hydrogen bonds to G6-O6 and is close to G6-N7, with distance $\mathrm{H}(\mathrm{N}) \ldots \mathrm{N}(\mathrm{G})$ of $3.4 \AA \pm 0.2$. In the same complex, with the carboxamide group in the trans orientation, the $N, N$ dimethylammonium group forms two hydrogen bonds to G6-N7/O6 atoms, a weak and a strong, with distances of $3.3 \pm 0.2$ and $2.1 \AA \pm 0.2$, respectively. 
In the molecular dynamic simulation of the (6)-CGCGAATTCGCG complex, with the carboxamide group in the cis orientation, the protonated $\mathrm{N}, \mathrm{N}$-dimethylamino group forms strong hydrogen bond to G2-O6. In the same complex, with the carboxamide group in the trans orientation, the protonated $N, N$-dimethylamino group forms strong hydrogen bond to G2-O6 and is in close contact with G2-N7, with distance $\mathrm{H}(\mathrm{N}) \ldots \mathrm{N}(\mathrm{G})$ of $3.6 \AA \pm 0.3$.

In the molecular dynamic simulation of the (7)-CCGGCGCCGG complex, with the carboxamide group in the cis orientation, the protonated $N, N$-dimethylamino group makes strong hydrogen bonding interaction with G6-O6 and is close to G6-N7, with distance $\mathrm{H}(\mathrm{N}) \ldots \mathrm{O}(\mathrm{G})$ of $3.8 \AA \pm 0.2$. In the same complex, with the carboxamide group in the trans orientation, the protonated $\mathrm{N}, \mathrm{N}$-dimethylamino group strongly hydrogen bonds to G6-O6, with distance $\mathrm{H}(\mathrm{N}) \ldots \mathrm{O}(\mathrm{G})$ of $1.9 \AA \pm 0.2$.

In the molecular dynamic simulation of the (7)-CGCGAATTCGCG complex, with the carboxamide group in the $c i s$ orientation, the $N, N$-dimethylammonium group gives a good hydrogen bond to G2-O6, with distance $\mathrm{H}(\mathrm{N}) \ldots \mathrm{N}(\mathrm{G})$ of $2.1 \AA \pm 0.3$. In the same complex, with the carboxamide group in the trans orientation, the protonated $N, N$-dimethylamino group makes a strong hydrogen bond solely to $\mathrm{O} 6$ of guanine-2, with distance $\mathrm{H}(\mathrm{N}) \ldots \mathrm{O}(\mathrm{G})$ of $1.9 \AA$ \pm 0.1 .

\section{Discussion}

We have found by molecular mechanics methods that in the, phenazine, azacridine, pyridoquinoxaline and acridine-4-carboxamide series 1, 4-7 the $N, N$-dimethylaminoethyl side chain does interact with guanine of two oligonucleotides in M1 orientations, with the 4carboxamide group rotated cis or trans with respect to the ring nitrogen. By contrast, when the side chain is peri to a $\mathrm{CH}$ group, as in the anthracene and acridine-1-carboxamide compounds, $\mathbf{2}$ and $\mathbf{3}$, the side chain fails to make this interaction. An important difference between two ligand classes is that, like 9-amino-DACA, compound $\mathbf{1}$, the carboxamide groups of compounds 4-7 can lie in the plane of the chromophore, whereas in the case of the anthracene and acridine-1-ligands, steric interaction with the peri hydrogen atom prevents the carboxamide from being coplanar. Thus, it is obvious that coplanarity of the carboxamide and chromophore is a prerequisite for effective side chain-guanine interactions among the linear tricyclic carboxamides such as DACA and its analogues. 
However, when the distance between the 4-carboxamide and $N, N$-dimethylamino group is lengthened (compounds 11 and 12), the interaction with guanine is lost probably due to the internal hydrogen bond between the carbonyl oxygen and the protonated $N, N$-dimethylamino group.

In the cases of the oxopyridoquinoxaline and quinoline ligands, $\mathbf{8}$ and $\mathbf{1 3}$, the existence of the internal hydrogen bond between the carbonyl oxygen and the protonated $N, N$-dimethylamino group seems to prevent the $N, N$-dimethylaminoethyl side chain to interact with guanine.

In the case of the phenoxazine ligand $\mathbf{9}$, the carbonyl oxygen forms an internal hydrogen bond in the cis configuration with the peri $\mathrm{NH}$ atom, but the existence of a second internal hydrogen bond between the carbonyl oxygen and the protonated $N, N$-dimethylamino group seems to prevent the $N, N$-dimethylaminoethyl side chain to interact with guanine. Clearly, for this ligand the trans carboxamide orientation is prohibited by steric collision of the two $\mathrm{NH}$ groups, which will force an angular conformation between chromophore and carboxamide. In the case of the xanthenone ligand 10, the $\mathrm{NH}$ of the carboxamide forms an internal hydrogen bond in the trans configuration with the peri oxygen atom, but the existence of a second internal hydrogen bond between the carbonyl oxygen and the protonated $N, N$-dimethylamino group also seems to prevent the $N, N$-dimethylaminoethyl side chain to interact with guanine. Owing to this internal hydrogen bond, the cis xanthenone carboxamide configuration should be unable to bond successfully with the guanine O6/N7 atoms.

From an assessment of the above data regarding tricyclic carboxamides, prerequisite for the interaction between protonated $N, N$-dimethylaminoethyl moiety and guanine is the formation of only one internal hydrogen bond between carboxamide and peri $\mathrm{NH}+$ in the case of 9amino-DACA (1) or peri $\mathrm{N}$ in the cases of DACA (4), azacridine (5), phenazine (6) and pyridoquinoxaline (7). The presence of an additional internal hydrogen bond between oxygen carboxamide and protonated $N, N$-dimethylamino group in the cases of tricyclic systems bearing peri $\mathrm{NH}$ (phenoxazine, 9) or $\mathrm{O}$ (xanthenone, 10) group, prevents the interaction between side chain and guanine. Also, the formation of one internal hydrogen bond between oxygen carboxamide and protonated $N, N$-dimethylamino group inhibits the interaction between side chain and guanine in the case of acridine-1-carboxamide (3). It is remarkable, that our findings correlate well with reported results obtained from the kinetic studies of the binding of such tricyclic carboxamides to DNA. According to Wakelin et al the complexes of 1, 4 and 6 dissociate from calf thymus DNA by a kinetic pathway involving four discernible 
steps, indicating that they interact favorably with the guanine O6/N7 atoms. By contrast, the tricyclic carboxamides $2,3,9,10,11$ and 12 with neutral chromophore dissociate from DNA by a different mechanism in which it appears their side chain fail to interact with guanine. There is no data available in the literature regarding the kinetics of compounds $\mathbf{5}, \mathbf{7 , 8}$ and 13 with DNA and therefore correlation with our results is not possible.

In all the examined complexes of compounds 1 and 4-7 with the oligonucleotides, the distance between the charged amino group and O6 guanine base is rather constant i.e. from 1.8 to $2.2 \AA$, indicating the presence of a hydrogen bond that remains stable during the entire molecular dynamic simulation. The deviations in kinetic, potential energy and in the angles of hydrogen bonds were small. Based on the above observations, we can assume that the complexes do not experience large conformational changes during the sampling time with respect to the initial structure.

Finally, based on the literature [6] and our molecular modeling experiments we can assume that there is a broad positive correlation between cytotoxicity of the protonated $\mathrm{N}, \mathrm{N}$ dimethylamino carboxamides with neutral chromophores and their interaction to guanine, with the compounds 4, 5 and $\mathbf{6}$ with only one internal hydrogen bond between carboxamide and peri nitrogen being potent cytotoxins. The compounds 11 and 12 (with two internal hydrogen bonds: one between carboxamide and peri nitrogen and the second between carbonyl oxygen and protonated $N, N$-dimethylamino group) are also potent cytotoxins. The compounds 2 (with no internal hydrogen bonds), $\mathbf{3}$ (with one internal hydrogen bond between carbonyl oxygen and protonated $N, N$-dimethylamino group) and the compounds $\mathbf{9}$ and $\mathbf{1 0}$ (with two internal hydrogen bonds: one between carboxamide and peri heteroatom and the second between carbonyl oxygen and protonated $N, N$-dimethylamino group) are less cytotoxic. The compound $\mathbf{1 3}$ (with two internal hydrogen bonds: one between carboxamide and peri heteroatom and the second between oxygen carboxamide and protonated $\mathrm{N}, \mathrm{N}$ dimethylamino group) is inactive. In conclusion, prerequisite for the interaction between protonated $N, N$-dimethylaminoethyl moiety and guanine is the formation of only one internal hydrogen bond between carboxamide and peri $\mathrm{NH}+$ in the case of 9-amino-DACA or peri $\mathrm{N}$ in the cases of DACA, azacridine, phenazine and pyridoquinoxaline. The presence of an additional internal hydrogen bond between oxygen carboxamide and protonated $\mathrm{N}, \mathrm{N}-$ dimethylamino group in the cases of tricyclic systems bearing peri $\mathrm{NH}$ or $\mathrm{O}$ group, prevents the interaction between side chain and guanine and leads to reduction of the cytotoxicity. Additionally, the present molecular dynamics simulations show that the compounds 1 and 4-6 
are capable of forming rather stable DNA complexes with small deviations in potential energy, RMSD and in the angles of hydrogen bonds, which is in accordance with good in vitro activities, further supported by in vivo experiments. 


\section{References}

1. Varvaresou A, Tsotinis A, Papadaki-Valiraki A, Siatra-Papastaikoudi Th (1996) Bioorg Med Chem Lett 6:861-864

2. Kapadia GJ, Tokuda H, Shridhar R, Balasubramanian V, Takayasu J, Bu P, Enjo F, Takasaki M, Konoshima T, Nishino H (1998) Cancer Lett 129:87-95

3. Varvaresou A, Iakovou K, Gikas E, Fichtner I, Fiebig HH, Kelland LR, Double JA, Bibby MC, Hendriks HR (2004) Anticancer Res 24:907-920

4. Varvaresou A, Tsirivas E, Tsaoula E, Protopapa E (2005) Rev Clin Pharmacol Pharacokin 19:11-16

5. Miller KJ, Newlin DD (1982) Biopolymers 21:633-652

6. Wakelin LPG, Adams A, Denny WA (2002) J Med Chem 45:894-901

7. Atwell GJ, Rewcastle GW, Baguley BC, Denny WA (1987) J Med Chem 30:664-669

8. Schneider E, Darkin SJ, Lawson PA, Ching LM, Ralph RK, Baguley BC (1988) Eur J Cancer Clin Oncol 24:1783-1790

9. Baguley BC, Zhuang L, Marshall E (1995) Cancer Chemother Pharmacol 36:244-248

10. Finlay GJ, Riou JF, Baguley BC (1996) Eur J Cancer A 32A:708-714

11. Atwell GJ, Cain BF, Baguley BC, Finlay GJ, Denny WA (1984) J Med Chem 27:14811485

12. Denny WA, Roos IAG, Wakelin LPG (1986) Anticancer Drug Des 1:141-147

13. Rewcastle GW, Atwell GJ, Chambers D, Baguley BC, Denny WA (1986) J Med Chem 29:472-477

14. Denny WA, Atwell GJ, Rewcastle GW, Baguley BC (1987) J Med Chem 30:658-663

15. Wakelin LPG, Atwell GJ, Rewcastle GW, Denny WA (1987) J Med Chem 30:855-861

16. Adams A, Guss JM, Collyer CA, Denny WA, Wakelin LPG (1999) Biochemistry 38:9221-9233

17. Adams A, Guss JM, Collyer CA, Denny WA, Prakash AS, Wakelin LPG (2000) Mol Pharmacol 58:649-658

18. Todd AK, Adams A, Thorpe JH, Denny WA, Wakelin LPG, Cardin CJ (1999) J Med Chem 42:536-540

19. Thorpe JH, Hobbs JR, Todd AK, Denny WA, Charlton P, Cardin CJ (2000) Biochemistry 39:15055-15061

20. Adams A, Guss JM, Collyer CA, Denny WA, Wakelin LPG (2000) Nucleic Acids Res $28: 4244-4253$ 
21. Yang Xl, Robinson H, Gao YG, Wang AHJ (2000) Biochemistry 39:10950-10957

22. Setzer WN, Rozmus GF, Setzer MC, Schmidt JF, Vogler B., Reeb S., Jackes BR, Irvine AK (2006) J Mol Model 12:703-711

23. Byler KG, Wamg C, Setzer WN (2009) J Mol Model 15:1417-1426

24. Loza-Mejía MA, Castillo R, Lira-Rocha A (2009) J Mol Graph Model 27:900-907

25. Braña MF, Cacho M, García MA, Pascual-Tereza B, Ramos A, Acero N, Llinares F, Muñoz-Mingarro D, Abradelo C, Rey-Stolle MF, Yuste M (2002) J Med Chem 45:58135816

26. Braña MF, Cacho M, García MA, Pascual-Tereza B, Ramos A, Dominguez MT, Pozuelo JM, Abradelo C, Rey-Stolle MF, Yuste M, Báñez-Coronel M, Lacal JC (2004) J Med Chem 47:1391-1399

27. Chen Q, Deady LW, Baguley BC, Denny WA (1994) J Med Chem 37:593-597

28. Varvaresou A, Iakovou K (2002) J Heterocycl Chem 39:1173-1176

29. Atwell GJ, Bos CD, Baguley BC, Denny WA (1988) J Med Chem 31:1048-1052

30. Müler W, Crothers DM, Thara T (1975) Eur J Biochem 54:267-277

31. Bailly C, Denny WA, Mellor LE, Wakelin LPG, Waring MJ (1992) Biochemistry 31:3514-24

32. Fischer G, Pindur U (1999) Pharmazie 54: 3-93

33. Wadkins RM, Graves DE (1991) Biochemistry 30:4277-4283

34. Krugh TR (1994) Curr Opin Struct Biol 4:351-364

35. Krugh TR, Reinhardt CG (1975) J Mol Biol 97:133-162

36. Hyperchem, Release 7.5 (2003) Hypercube Inc

37. Spartan 04, 1991-2003, Wavefunction Inc

38. Krieger E, Koraimann G, Vriend G (2002) Proteins 47:393-402

39. Krieger E, Darden T, Nabuurs SB, Finkelstein A, Vriend G (2004) Proteins 57:678-683

40. Kawata M, Nagashima U (2001) Chem Phys Lett 340:165-172 
Tables

Table 1 Hydrogen bonds* of a) N, N-dimethylammonium group of compounds 1-13 with Guanine-O6/N7or the carboxamide moiety and b) the carboxamide moiety with chromophore

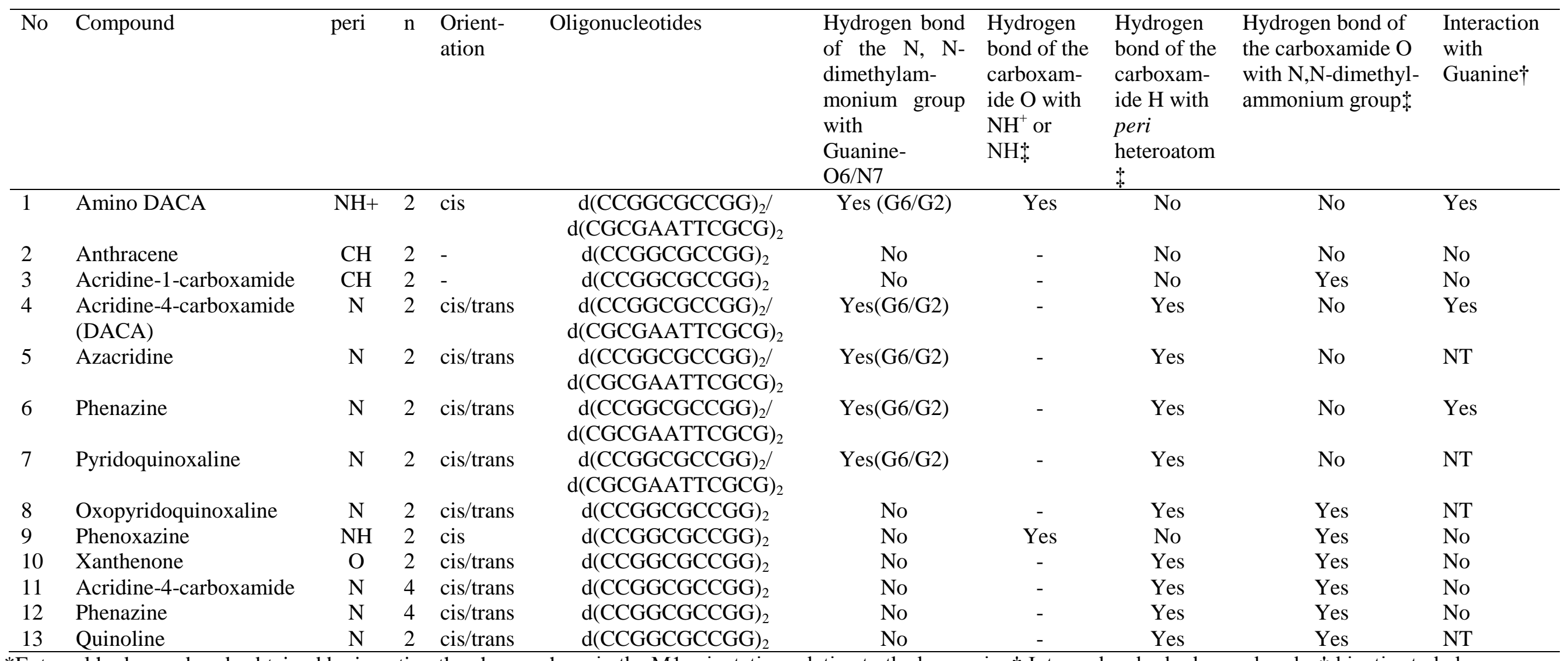

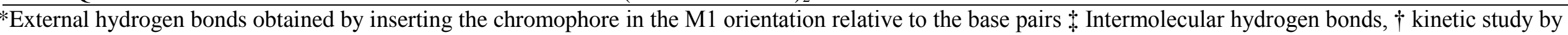

Wakelin et al, NT=Not tested 


\section{Figure captions}

Fig. 1 Structures of compounds studied

Fig. 2 Schematic view from the major groove of the four complexes between compounds 1-13 and the central $\mathrm{CG}$ of $\mathrm{d}(\mathrm{CCGGCGCCGG})_{2}$. The white square in the chromophore represents the orientation of the ring with the side chain, and the sphere represents the protonated dimethylamino group from the side chain. Left : complexes with the linker in the major groove and the ring with the side chain stacked between CG (M1), or between GC (M2). Right : complexes with the linker in the minor groove and the ring with the side chain stacked between $\mathrm{CG}(\mathbf{m} \mathbf{1})$, or between GC (m2)

Fig. 3 Plots of the variation of the rmsd of compounds 1 and 4-7 throughout the simulation

Fig. 4 Plots of the variation of the potential energy of compounds 1 and 4-7 throughout the simulation

Fig. 5 Structures of compounds 2, 3, 7 and $\mathbf{8}$ after geometry optimization by Hartree-Fock /6-31G* calculations. Dotted lines indicate hydrogen bonds

Fig. 6 Graphic representation of DACA-CCGGCGCCGG model in the initial state, and after 5,10 and 15 ns simulation. Hydrogen are omitted for clarity. The figures show that the structure has remained stable for the duration of the simulation, without incurring large conformational changes 


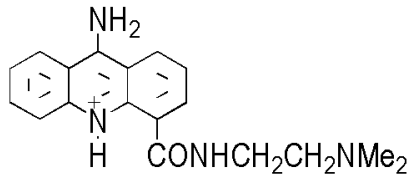

1

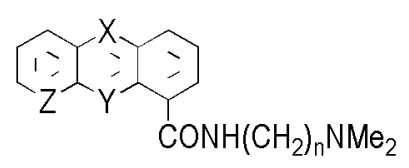

2-12

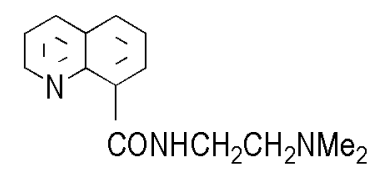

13

2. $X, Y, Z=C H, n=2$

3. $X=N, Y, Z=C H, n=2$

4: $X, Z=C H, Y=N, n=2$

5: $X=C H, Y, Z=N, n=2$

6: $X, Y=\mathrm{N}, Z=\mathrm{CH}, \mathrm{n}=2$

7: $X, Y, Z=N, n=2$

8: $X=N O, Y, Z=N, n=2$

9: $X=0, Y=N H, Z=C H, n=2$

10: $X=\mathrm{CO}, Y=\mathrm{O}, Z=\mathrm{CH}, \mathrm{n}=2$

11: $X, Z=\mathrm{CH}, Y=N, n=4$

12: $X, Y=N, Z=C H, n=4$ 

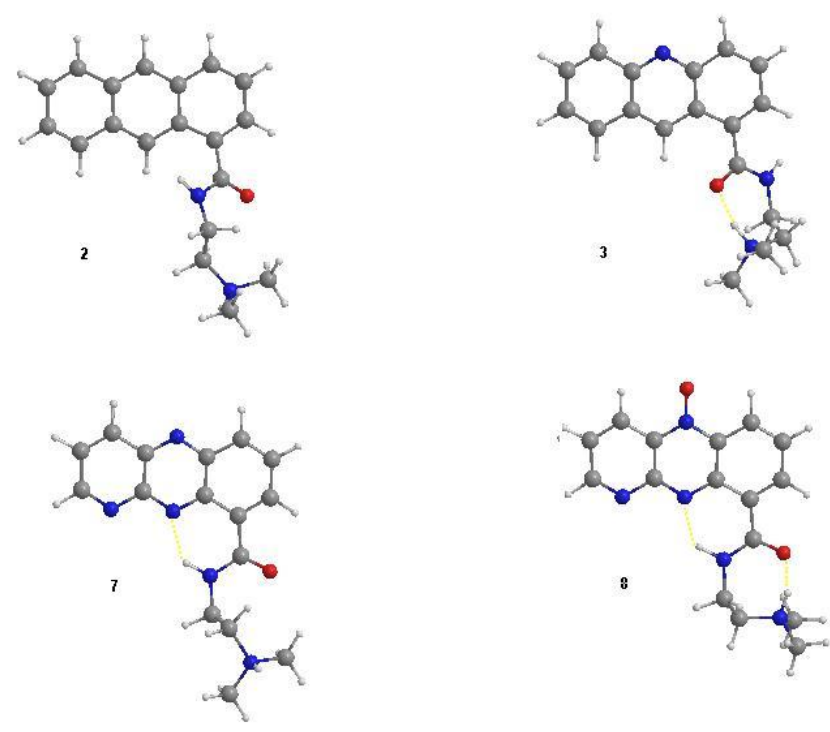


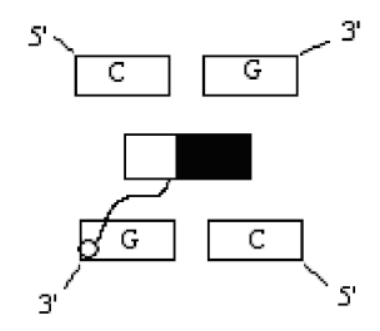

$\mathrm{MI}$
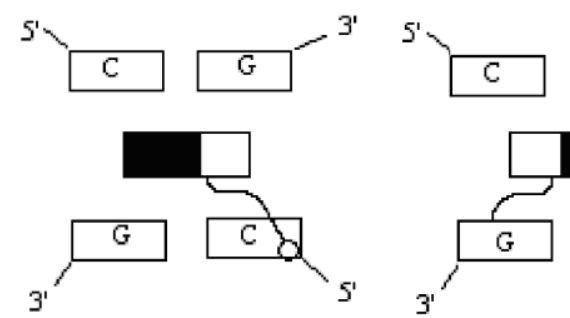

M2
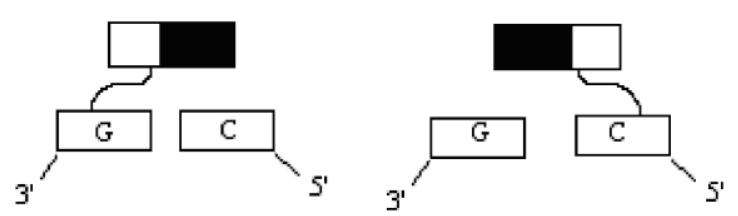

$\mathrm{ml}$

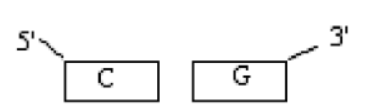

Compounds 1-13

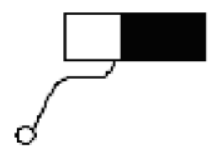




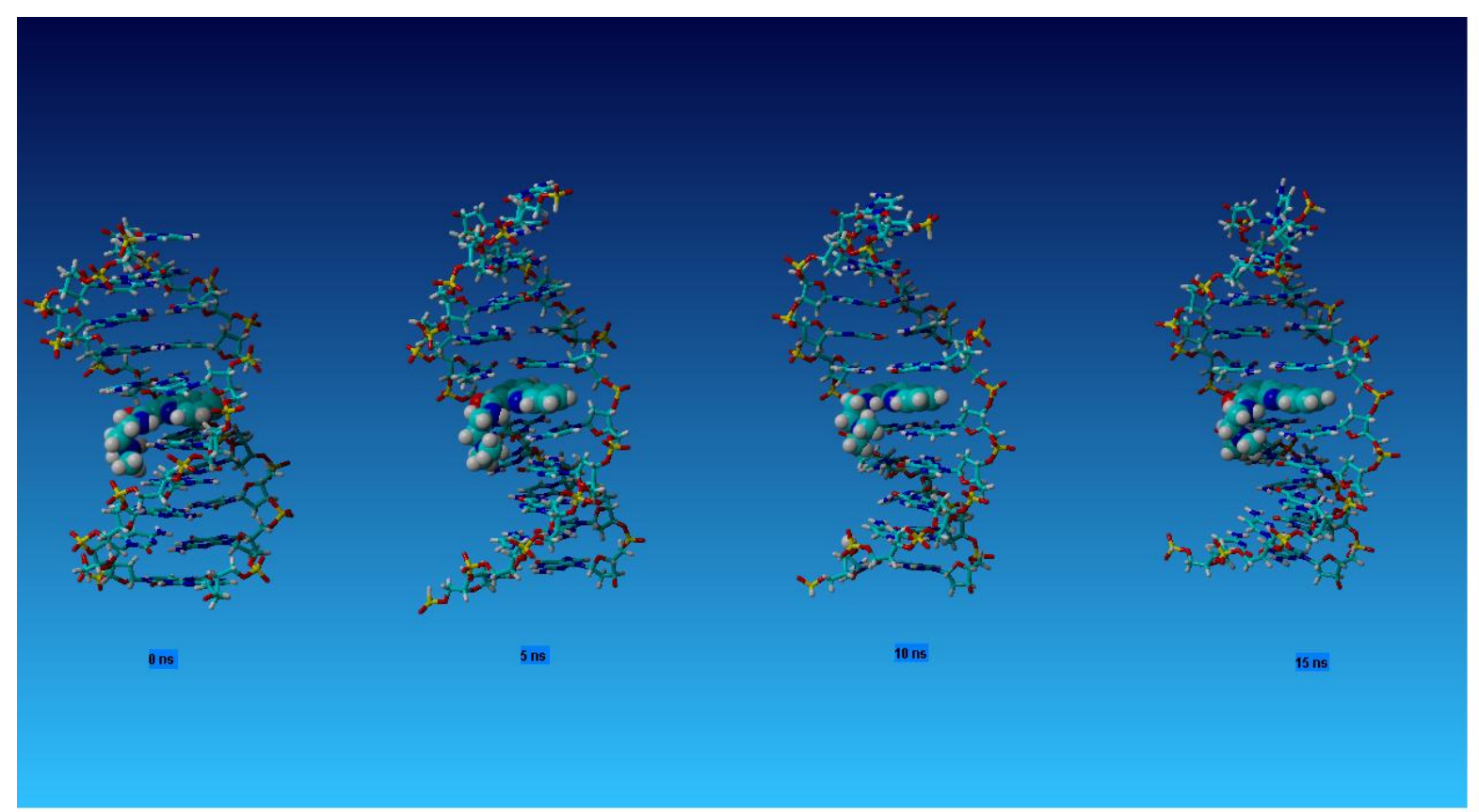




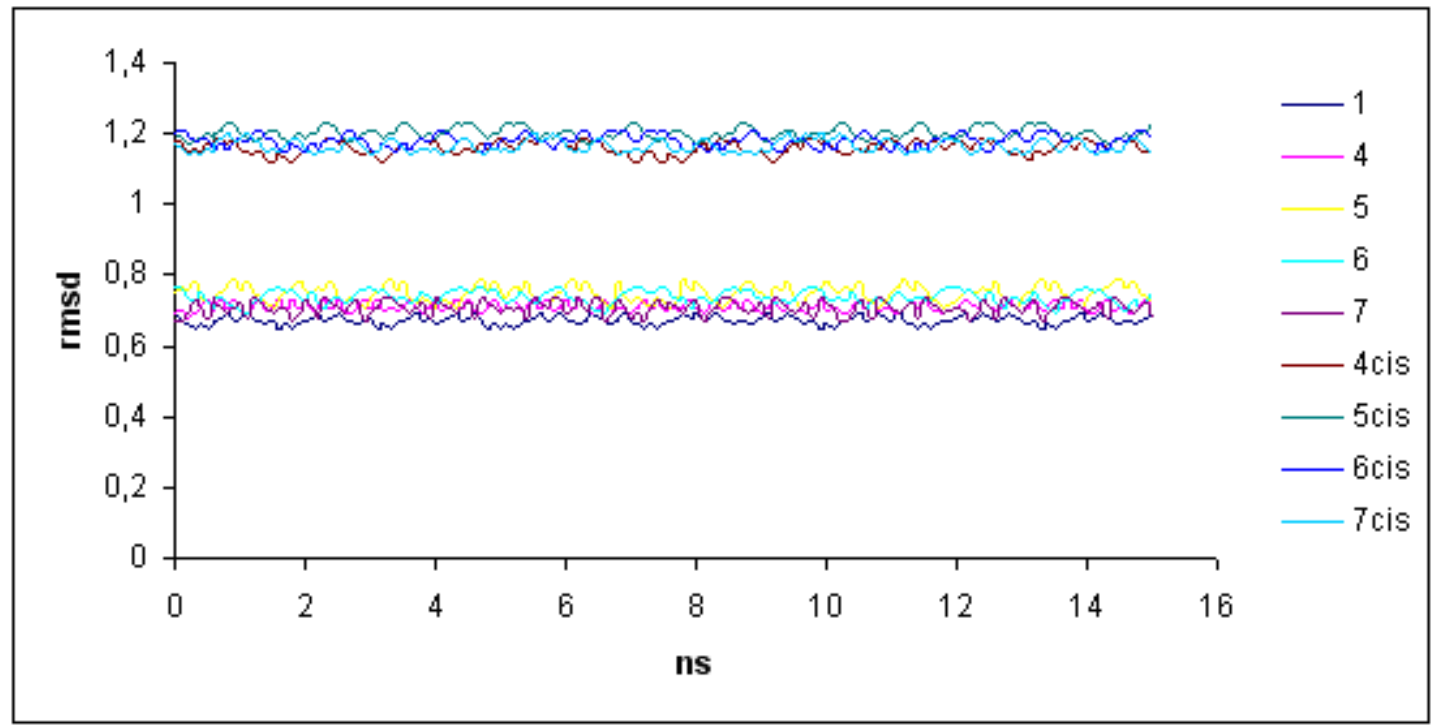




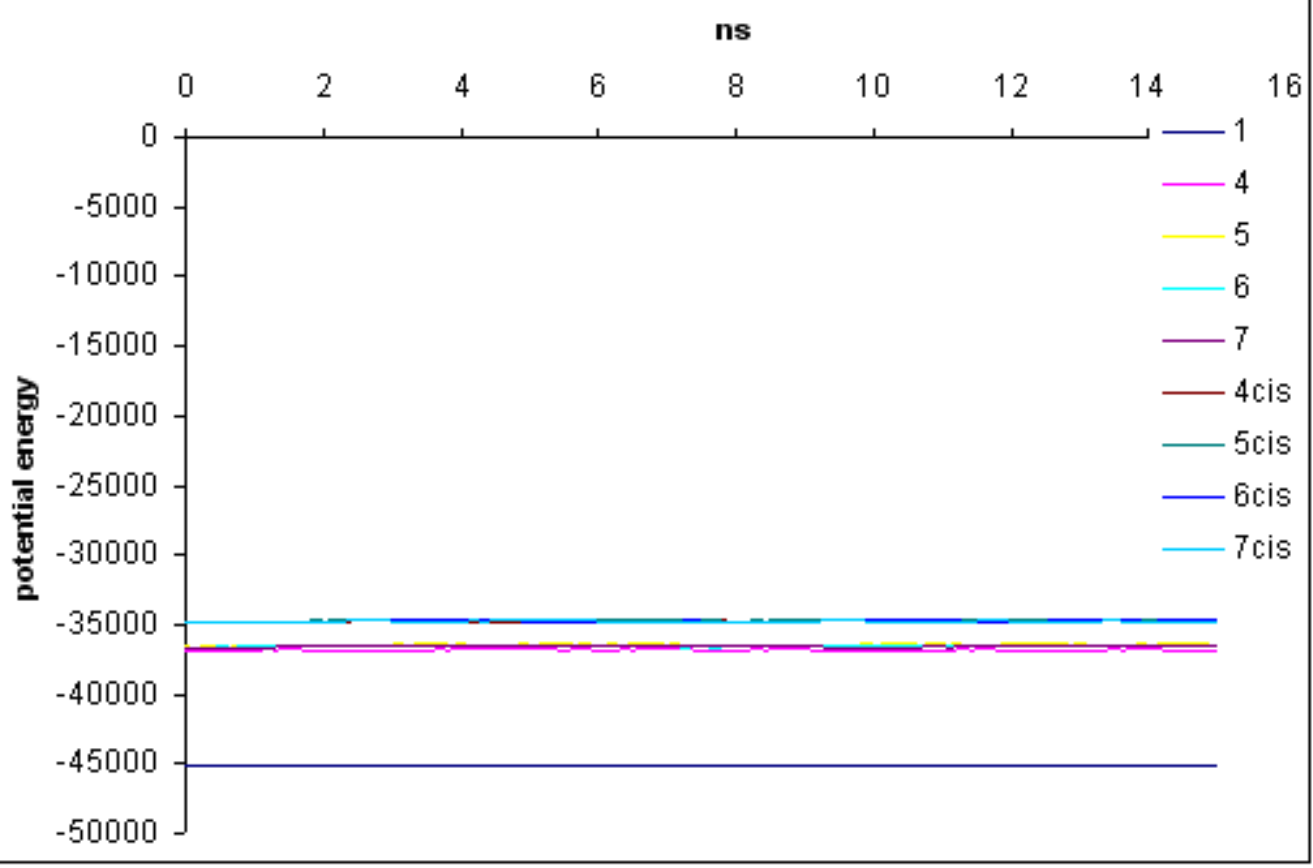




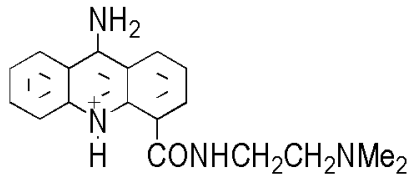

1

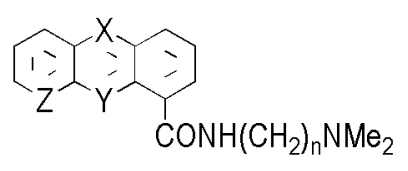

2-12

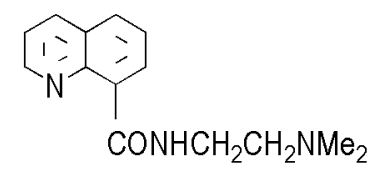

13

2. $X, Y, Z=C H, n=2$

3. $X=N, Y, Z=C H, n=2$

4: $X, Z=C H, Y=N, n=2$

5: $X=C H, Y, Z=N, n=2$

6: $X, Y=\mathrm{N}, Z=\mathrm{CH}, \mathrm{n}=2$

7: $X, Y, Z=N, n=2$

8: $X=N O, Y, Z=N, n=2$

9: $X=0, Y=N H, Z=C H, n=2$

10: $X=\mathrm{CO}, Y=\mathrm{O}, Z=\mathrm{CH}, \mathrm{n}=2$

11: $X, Z=\mathrm{CH}, Y=N, n=4$

12: $X, Y=N, Z=C H, n=4$ 